\title{
Therapeutic Anticoagulation After Craniotomies: Is the Risk for Secondary Hemorrhage Overestimated?
}

\author{
Christian Scheller $^{1}$ Jens Rachinger ${ }^{1}$ Christian Strauss ${ }^{1} \quad$ Alex Alfieri $^{1} \quad$ Julian Prell ${ }^{1} \quad$ Gershom Koman $^{1}$ \\ ${ }^{1}$ Department of Neurosurgery, University of Halle-Wittenberg, Halle, \\ Germany \\ J Neurol Surg A 2014;75:2-6. \\ Address for correspondence Christian Scheller, MD, Department of \\ Neurosurgery, University of Halle-Wittenberg, Ernst-Grube-Str. 40, \\ Halle (Saale), Sachsen-Anhalt 06097, Germany \\ (e-mail: christian.scheller@uk-halle.de).
}

\begin{abstract}
Objective Deep venous thrombosis (DVT) and pulmonary embolism (PE) are major causes of postoperative morbidity and mortality in surgery. However, there is neither a standardized protocol for perioperative prevention of DVT or PE in neurosurgery nor a consensus concerning the management of postoperative DVT or PE after craniotomy in the early postoperative course.

Methods We retrospectively analyzed management and complications in a group of patients with postoperative DVT or PE after craniotomy between 2006 and 2011 to estimate the risk of secondary hemorrhage under therapeutic anticoagulation. The interval between time of craniotomy and diagnosis of PE or DVT, administered anticoagulation, and the appearance of a clinically relevant secondary hemorrhage were analyzed.

Results Forty-two patients met the given criteria. Indications for surgery were intracranial tumors $(n=33)$, aneurysms $(n=5)$, and hematomas $(n=4)$. PE or DVT was observed between the first and the 28th postoperative day (median, fifth postoperative day). Therapeutic anticoagulation was performed with enoxaparin or heparin (according to partial thromboplastin time levels). Full heparinization was applied in 30 patients between the second and the 30th postoperative day (median,

\section{Keywords}

- craniotomy

- therapeutic anticoagulation

- pulmonary embolism

- deep venous thrombosis 12th postoperative day). None of these patients developed a secondary hemorrhage. Conclusion The documented differences in the anticoagulative drug used, the drug's dosage, and the start of medication reflect the lack of a standardized protocol concerning the treatment of postoperative PE or DVT after craniotomy. A more aggressive management regarding the application of anticoagulative drugs after craniotomy may be justified considering the absence of clinically relevant hemorrhages in this study and the life-threatening potential of perioperative DVT or PE.
\end{abstract}

\section{Introduction}

After neurosurgical procedures with long general anesthesia, deep venous thrombosis (DVT) and pulmonary embolism (PE) are major causes of postoperative morbidity and mortality. In untreated control groups of randomized studies after

received

July 12,2012

accepted after revision

January 14, 2013

published online

August 19, 2013 craniotomies, DVT rates exceed $25 \%{ }^{1}$ In such patients, clinically evident DVT occurs in $2 \%$ to $4 \%{ }^{2}$ PE rates vary between $0.8 \%$ and $2 \%{ }^{3,4}$ The efficiency, cost effectiveness, and safety of prophylactically administered anticoagulative drugs after general surgical and neurosurgical procedures have been demonstrated in clinical studies. ${ }^{5-10}$ Furthermore, the (c) 2014 Georg Thieme Verlag KG Stuttgart · New York
DOI http://dx.doi.org/ 10.1055/s-0033-1345686. ISSN 2193-6315. 
additive use of mechanical devices such as elastic graduated compression stockings or pneumatic compression boots has been shown to reduce the incidence of DVT after surgical procedures. ${ }^{11}$ However, a standardized protocol in the perioperative prevention of DVT for neurosurgical procedures is still missing. ${ }^{12}$ For one, this is because of the fear of secondary hemorrhage, which is supposed to be increased when applying anticoagulative drugs early in the postoperative course. Furthermore, the available literature does not yield a consensus concerning the management of postoperative DVT or PE with therapeutic anticoagulation after craniotomies.

\section{Methods}

A consecutive series of patients undergoing craniotomies between 2006 and 2011 with postoperative PE or DVT was retrospectively analyzed regarding demographic attributes, diagnosis, method of perioperative thrombosis prevention, and interval between surgery and PE or DVT. PE and DVT were detected by elevated D-dimer levels, ultrasonography, and lung computed tomography (CT). In addition, the administered anticoagulative medication and its dosage were examined as well as patients' weight and kidney and liver functions. Secondary hemorrhage was excluded either by uneventful course or by cranial CT. Routine postoperative CT was not performed. The decision for postoperative CT was made individually and after consultation with the surgeon. The surgeon decided on the dosage of the applied anticoagulative drugs depending on the assumed risk for a secondary hemorrhage in each individual case. Subtherapeutic anticoagulation was defined as a dosage higher than prophylactic in contrast to therapeutic heparinization as the standard dosage for treatment of PE or DVT in a nonsurgical setting.

\section{Results}

A consecutive series of 42 patients was retrospectively analyzed. Indications for surgery were meningiomas $(n=17)$, vestibular schwannomas $(n=8)$, aneurysms $(n=5)$, gliomas $(n=4)$, subdural hematomas $(n=3)$, ependymoma $(n=1)$, hemangioblastoma $(n=1)$, brain metastases $(n=1)$, prolactinoma $(n=1)$, and intracerebral hemorrhage $(n=1)$. A total of 26 patients were female, and 16 were male. Patients' age ranged from 17 to 80 years (mean, 55 years). None of the patients had a severe impairment of kidney or liver function.

Prevention of postoperative thromboembolism was performed with elastic graduated compression stockings and enoxaparin, certoparin, or unfractionated heparin (UFH). Low-molecular-weight heparin (LMWH) for standard perioperative prophylaxis was given starting on the first postoperative day. In some cases of, for example, intracerebral or subdural hematoma, the start of LMWH administration was determined individually. None of the patients received anticoagulative drugs on the day of surgery. Prophylactic medication was started on the first postoperative day in 33 patients, on the second postoperative day in two patients, on the third postoperative day in three patients, on the tenth

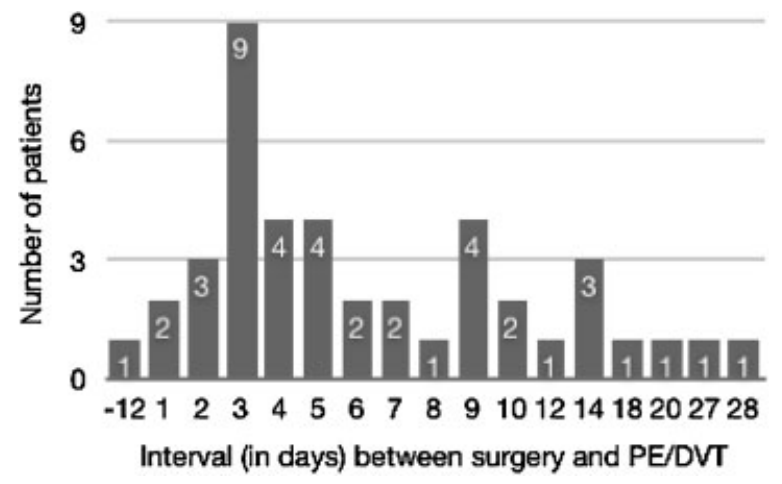

Fig. 1 Interval between surgery and diagnosis of pulmonary embolism (PE) or deep venous thrombosis (DVT).

postoperative day in one patient, on the eleventh postoperative day in two patients, and on the twelfth postoperative day in one patient.

Postoperative PE or DVT was observed in 41 patients between the first and the 28th postoperative day (median, fifth postoperative day) (-Fig. 1). Seven patients suffered from PE and DVT, one patient only from PE, and 34 patients exclusively from DVT. In one patient (number 9) a DVT 12 days before surgery was diagnosed, resulting in higher pre- and postoperative dosages of enoxaparin ( $\mathbf{- F i g . 1}$ ).

During the postoperative course, 19 patients developed symptoms of DVT or PE (dyspnea, pain or swelling in the lower leg), and 23 did not have any symptoms. In these 23 patients, pre- and postoperative $\mathrm{D}$-dimer levels were measured according to a protocol of a separate study investigating postoperative $D$-dimer values. In case of distinct postoperative elevation of D-dimer levels, deep vein ultrasonography or lung CT scans were performed to verify PE or DVT.

After the diagnosis of postoperative PE or DVT, the dosage of enoxaparin or partial thromboplastin time (PTT)-monitored UFH was increased in 39 patients. Prophylactic anticoagulation was continued in the three remaining patients because of the small extent of DVT. Therapeutic heparinization was applied to 30 of the 42 patients.

Assuming that the risk for a secondary hemorrhage is higher in the early postoperative course, ${ }^{12}$ the first week after surgery was analyzed in detail. During the first 7 days after surgery (starting on postoperative day 2 to 7), 20 patients were treated with higher than prophylactic dosages. Seven of them received anticoagulative drugs in dosages of standard therapeutic heparinization, and 13 patients received subtherapeutic dosages between prophylactic and therapeutic heparinization. For anticoagulation, LMWH (enoxaparin subcutaneously $2 \times 30 \mathrm{mg}$ to $2 \times 70 \mathrm{mg}$ ) or UFH (target PTT, 60-80 sec) delivered through a syringe pump were used (-Fig. 2). - Fig. 2 illustrates the management of anticoagulation in relation to the interval between craniotomy and start of therapeutic/subtherapeutic heparinization. Lung CT scans revealed extended bilateral PE in 5 of these 20 patients. The remaining 15 patients exclusively had DVT.

None of the 42 patients had a secondary hemorrhage after LMWH or heparin administration. Secondary hemorrhage 


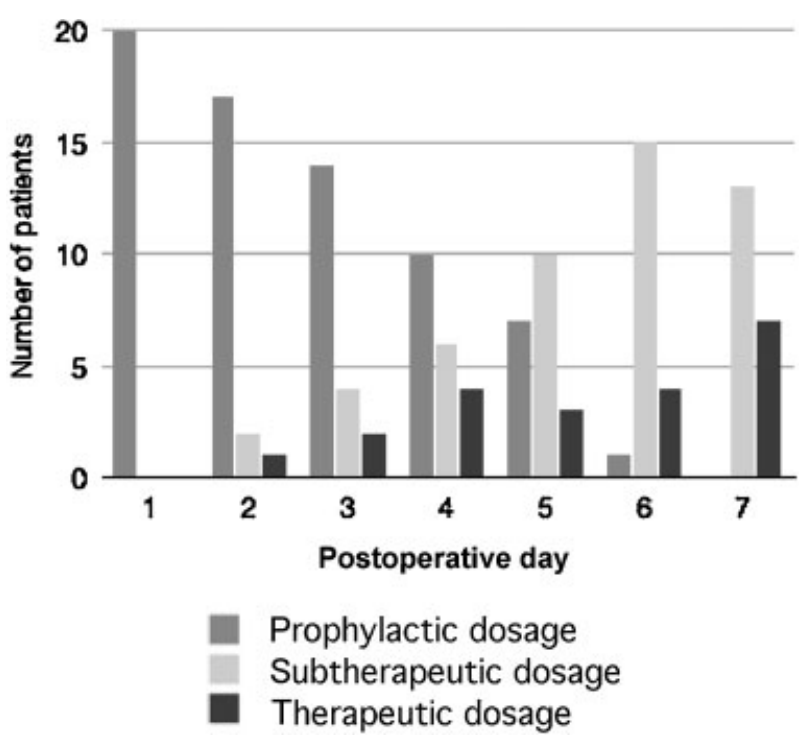

Fig. 2 Dosage distribution in the 20 patients receiving higher than prophylactic dosages before postoperative day 7 .

was excluded either by a clinical uneventful course $(n=14)$ or by cranial CT $(n=28)$. In the 28 patients undergoing cranial $\mathrm{CT}$, the first postoperative cranial $\mathrm{CT}$ scan was performed on the day of surgery or in the first 2 postoperative days, and additional CT scans followed in the further clinical course. In 13 of these 28 patients, the cranial CT scans showed blood in the resection area before therapeutic anticoagulation was begun. Higher than prophylactic dosages were administered despite these findings, and no increase of hemorrhage in further CT scans or clinical deterioration in the further course was observed.

\section{Discussion}

The general risk for secondary hemorrhage after intracranial procedures was reported at $1.5 \%$ to $2.5 \%{ }^{13}$ Administration of heparin 12 hours after intracranial surgery is relatively safe. ${ }^{14-16}$ In contrast, administration of enoxaparin in prophylactic dosages during induction of anesthesia was associated with an increased risk for secondary hemorrhage after intracranial tumor surgery. ${ }^{17}$ Consequently, the interval between surgery and the start of anticoagulative medication seems to be more important than its dosage regarding the risk for secondary hemorrhage. In a prospective, randomized, double-blind study, there was no significant difference concerning hemorrhage complications between patients treated with subcutaneous heparin and those who were not. ${ }^{18}$ In contrast, a higher but not statistically significant hemorrhage complication rate $(2.5 \%$ vs. $0.8 \%)$ was reported for the prophylactic use of LMWH. ${ }^{19} \mathrm{~A}$ randomized pilot study showed no differences in postoperative hemorrhage, DVT, or PE between the group treated with subcutaneous heparin and the group with LMWH medication. ${ }^{2}$ Prophylactically administered LMWH starting within 24 hours after intracranial procedures reveals a risk reduction of $28.9 \%$ for DVT and of $40.2 \%$ for PE. ${ }^{19}$ Enoxaparin with compression stockings was more effective than compression stockings alone for prevention of DVT in elective neurosurgical procedures. ${ }^{20}$ Major hemorrhage complication rates of $3 \%$ were observed each in the enoxaparin and the placebo group. ${ }^{20}$ Cerrato et al reported a risk reduction from $34 \%$ to $6 \%$ for DVT by prophylactically administered heparin and no significantly increased rate of postoperative hematomas. ${ }^{21} \mathrm{~A}$ randomized, prospective, double-blind clinical trail revealed the efficacy and safety of enoxaparin and UFH for prophylaxis of venous thromboembolism after brain tumor surgery. ${ }^{22}$ However, heparininduced thrombocytopenia is a potential adverse drug effect of heparin medication with a reported frequency of $0.2 \%$ to $5 \%{ }^{23}$ After LWMH administration, the risk is five- to 10 -fold lower compared with UFH. ${ }^{24}$

A recently published study reviewed the current practice of perioperative prevention of DVT in German neurosurgical departments. ${ }^{12}$ There was no homogenous practice in the administration of heparin (UFH, LMWH, or both) and even compression stockings. In general, the risk for secondary hemorrhage under heparin was estimated higher after cranial versus spinal interventions. Heparin application after craniotomy starting during the first 5 days after surgery is assumed to be associated with a high or very high risk for relevant secondary hemorrhage by up to $20 \%$ of German neurosurgical departments. $^{12}$

Although a retrospective study cannot provide sufficient evidence of higher than prophylactic dosages of anticoagulative drugs being a safe treatment of PE or DVT after craniotomies, our results suggest that the rate of secondary hemorrhages under these circumstances may be overestimated. As in studies dealing with prophylactically administered low-dose heparin, ${ }^{10}$ no significant postoperative hemorrhages were observed in the present study analyzing therapeutic anticoagulation after craniotomies. Taking into consideration the potential risk for postoperative secondary hemorrhages, the risk-to-benefit ratio for using anticoagulative drugs in therapeutic dosages in patients with PE or DVT after craniotomy may be still favorable for the patient.

Our review of the literature and MEDLINE research revealed no further studies concerning therapeutic anticoagulation in cases of PE or DVT after craniotomies. In general, neurosurgeons hesitate to administer higher than prophylactic dosages of anticoagulative drugs in the early postoperative course after craniotomies. The reasons might be fear of secondary hemorrhages, the lack of clinical studies and accepted protocols in the postoperative management of PE and DVT after craniotomies and medicolegal considerations because LMWH and heparin are not approved after neurosurgical procedures.

The presented study is the first published clinical series of patients with LE or DVT after craniotomy receiving LMWH. The old data of Swann et $\mathrm{al}^{25}$ about management of LE or DVT lacks comparability because of the administered anticoagulative substances and the inclusion of patients with spinal disorders. The patient number in this study with DVT or LE after craniotomy was 10 , and the delay between surgery and beginning of anticoagulative medication was 8 to 54 days (mean, 26 days). Rebleeding was only observed in a patient 
without surgery. Ruff et $\mathrm{al}^{26}$ included more patients but used the same anticoagulative regimen as Swann et al but no LMWH. The prospective study of Gerlach et $\mathrm{al}^{27}$ reporting on 2823 patients undergoing major and minor intracranial procedures dealt with the prophylaxis of thromboembolic events. The safety of therapeutic heparin administration in patients diagnosed with DVT or PE in the course was not addressed, as in the study of Goldhaber et $\mathrm{al}^{22}$ and the review article of Epstein. ${ }^{28}$

In 14 patients in the present study, no postoperative CT scans were performed. These patients might have developed secondary hemorrhages. Yet without clinical signs, the radiologic finding of a small secondary hemorrhage would not have had any effects on treatment. Additionally, although limited by the variety of applied substances and their dosages, our data suggest no differences between the used anticoagulative drugs concerning the risk for rebleeding. Consequently, systematic studies are needed to estimate the risk for secondary hemorrhages under therapeutic anticoagulation after craniotomy.

\section{Conclusion}

The documented differences in the used anticoagulative substance, the start of medication, and the dosage reflect the need for a standardized protocol concerning the treatment of postoperative PE or DVT after craniotomy. Considering there was no secondary hemorrhage in this study and that PE and DVT are potentially life-threatening complications, it may be justified to be more aggressive in the application of anticoagulative drugs after craniotomy. Our observations do not support the assumption of a very high risk for rebleeding with application of heparin in the early days after craniotomy.

\section{Acknowledgments}

We thank Christin Zöller for her secretarial contribution and assistance in literature review.

\section{Conflict of Interest}

None

\section{References}

1 Hamilton MG, Hull RD, Pineo GF. Venous thromboembolism in neurosurgery and neurology patients: a review. Neurosurgery 1994;34(2):280-296, discussion 296

2 Macdonald RL, Amidei C, Baron J, et al. Randomized, pilot study of intermittent pneumatic compression devices plus dalteparin versus intermittent pneumatic compression devices plus heparin for prevention of venous thromboembolism in patients undergoing craniotomy. Surg Neurol 2003;59(5):363-372, discussion 372-374

3 Black PM, Baker MF, Snook CP. Experience with external pneumatic calf compression in neurology and neurosurgery. Neurosurgery 1986;18(4):440-444

4 Inci S, Erbengi A, Berker M. Pulmonary embolism in neurosurgical patients. Surg Neurol 1995;43(2):123-128, discussion 128-129
5 Barnett HG, Clifford JR, Llewellyn RC. Safety of mini-dose heparin administration for neurosurgical patients. J Neurosurg 1977; 47(1):27-30

6 Collins R, Scrimgeour A, Yusuf S, Peto R. Reduction in fatal pulmonary embolism and venous thrombosis by perioperative administration of subcutaneous heparin. Overview of results of randomized trials in general, orthopedic, and urologic surgery. $\mathrm{N}$ Engl J Med 1988;318(18):1162-1173

7 Kakkar VV, Stringer MD. Prophylaxis of venous thromboembolism. World J Surg 1990;14(5):670-678

8 Oster G, Tuden RL, Colditz GA. A cost-effectiveness analysis of prophylaxis against deep-vein thrombosis in major orthopedic surgery. JAMA 1987;257(2):203-208

9 Salzman EW, Davies GC. Prophylaxis of venous thromboembolism: analysis of cost effectiveness. Ann Surg 1980;191(2): 207-218

10 Wen DY, Hall WA. Complications of subcutaneous low-dose heparin therapy in neurosurgical patients. Surg Neurol 1998; 50(6):521-525

11 Turpie AGG, Gallus AS, Beattie WS, Hirsh J. Prevention of venous thrombosis in patients with intracranial disease by intermittent pneumatic compression of the calf. Neurology 1977;27(5): 435-438

12 Rachinger JC, Koman G, Scheller C, Prell J, Rampp S, Strauss C. Practice in the perioperative prevention of deep vein thrombosis in German neurosurgical departments: is there a trend towards homogenization? Cent Eur Neurosurg 2011;72(3):115-119

13 Apuzzo MLJ. Brain Surgery. Complication Avoidance and Management. New York: Churchill Livingstone; 1993

14 Kleindienst A, Harvey HB, Mater E, et al. Early antithrombotic prophylaxis with low molecular weight heparin in neurosurgery. Acta Neurochir (Wien) 2003;145(12):1085-1090, discussion 1090-1091

15 Norwood SH, McAuley CE, Berne JD, et al. Prospective evaluation of the safety of enoxaparin prophylaxis for venous thromboembolism in patients with intracranial hemorrhagic injuries. Arch Surg 2002;137(6):696-701, discussion 701-702

16 Raabe A, Gerlach R, Zimmermann M, Seifert V. The risk of haemorrhage associated with early postoperative heparin administration after intracranial surgery. Acta Neurochir (Wien) 2001; 143(1):1-7

17 Dickinson LD, Miller LD, Patel CP, Gupta SK. Enoxaparin increases the incidence of postoperative intracranial hemorrhage when initiated preoperatively for deep venous thrombosis prophylaxis in patients with brain tumors. Neurosurgery 1998;43(5): 1074-1081

18 Constantini S, Kanner A, Friedman A, et al. Safety of perioperative minidose heparin in patients undergoing brain tumor surgery: a prospective, randomized, double-blind study. J Neurosurg 2001; 94(6):918-921

19 Nurmohamed MT, van Riel AM, Henkens CMA, et al. Low molecular weight heparin and compression stockings in the prevention of venous thromboembolism in neurosurgery. Thromb Haemost 1996;75(2):233-238

20 Agnelli G, Piovella F, Buoncristiani P, et al. Enoxaparin plus compression stockings compared with compression stockings alone in the prevention of venous thromboembolism after elective neurosurgery. N Engl J Med 1998;339(2):80-85

21 Cerrato D, Ariano C, Fiacchino F. Deep vein thrombosis and lowdose heparin prophylaxis in neurosurgical patients. J Neurosurg 1978;49(3):378-381

22 Goldhaber SZ, Dunn K, Gerhard-Herman M, Park JK, Black PM. Low rate of venous thromboembolism after craniotomy for brain tumor using multimodality prophylaxis. Chest 2002;122(6): 1933-1937

23 Cuker A, Cines D. How I treat heparin-induced thrombocytopenia (HIT). Blood 2012;199(10):2209-2218 
6 Therapeutic Anticoagulation After Craniotomies Scheller et al.

24 Martel N, Lee J, Wells PS. Risk for heparin-induced thrombocytopenia with unfractionated and low-molecular-weight heparin thromboprophylaxis: a meta-analysis. Blood 2005;106(8):2710-2715

25 Swann KW, Black PM, Baker MF. Management of symptomatic deep venous thrombosis and pulmonary embolism on a neurosurgical service. J Neurosurg 1986;64(4):563-567

26 Ruff RL, Posner JB. Incidence and treatment of peripheral venous thrombosis in patients with glioma. Ann Neurol 1983;13(3):334-336
27 Gerlach R, Scheuer T, Beck J, Woszczyk A, Seifert V, Raabe A. Risk of postoperative hemorrhage after intracranial surgery after early nadroparin administration: results of a prospective study. Neurosurgery 2003;53(5):1028-1034, discussion 1034-1035

28 Epstein NE. A review of the risks and benefits of differing prophylaxis regimens for the treatment of deep venous thrombosis and pulmonary embolism in neurosurgery. Surg Neurol 2005;64(4): 295-301, discussion 302 